Publisher: Taylor \& Francis \& Informa UK Limited, trading as Taylor \& Francis Group

Journal: Scandinavian Journal of Forest Research

DOI: $10.1080 / 02827581.2016 .1195441$

\title{
Combining Spatiotemporal Corridor Design for Reindeer Migration with Harvest Scheduling in Northern Sweden
}

Rachel St John ${ }^{1}$, Karin Öhman², Sándor F. Tóth ${ }^{3}$, Per Sandström ${ }^{4}$, Anu Korosuo $^{5}$, Ljusk Ola Eriksson ${ }^{6}$

${ }^{1}$ (Corresponding Author) Industrial \& Systems Engineering, University of Washington, Seattle, United States,rkrieg@u.washington.edu

${ }^{2}$ Department of Forest Resource Management, Swedish University of Agricultural Sciences, Umeå, Sweden, karin.ohman@slu.se

${ }^{3}$ School of Environmental and Forest Sciences, University of Washington, Seattle, United States, toths@u.washington.edu

${ }^{4}$ Department of Forest Resource Management, Swedish University of Agricultural Sciences, Umeå,Sweden, per.sandstrom@slu.se

${ }^{5}$ Ecosystems Services and Management Program, International Institute for Applied Systems Analysis, Laxenburg, Austria, korosuo@iiasa.ac.at

${ }^{6}$ Department of Forest Resource Management, Swedish University of Agricultural Sciences, Umeå, Sweden, ljusk.ola.eriksson@slu.se

\section{Acknowledgements}

This project was funded jointly by the Swedish University of Agricultural Sciences and the Precision Forestry Cooperative at the University of Washington. The work was also 
partially financed by the EU FP7 project Advanced multifunctional management of European mountain forests (ARANGE). We would also like to thank Holmen for the forest dataset.

\section{Combining Spatiotemporal Corridor Design for Reindeer Migration with Harvest Scheduling in Northern Sweden}

Reindeer husbandry and commercial forestry seek to co-exist in the forests of Northern Sweden. As interwoven as the two industries are, conflicts have arisen. Forest practices have reduced the distribution of lichen, the main winter diet for reindeer. Forest practices have also increased forest density, compromising the animals ability to pass through forested areas on their migration routes. In an attempt to reduce impacts on reindeer husbandry, we present a spatially explicit harvest scheduling model that includes reindeer corridors with user-defined spatial characteristics. We illustrate the model in a case study and explore the relationship between timber revenues and the selection and maintenance of reindeer corridors. The corridors are not only to include sufficient lichen habitat, but they are also supposed to ensure access for reindeer by connecting lichen areas with linkages that allow unobstructed travel. Since harvest scheduling occurs over a planning horizon, the spatial configuration of corridors can change from one time period to the next in order to accommodate harvesting activities. Our results suggest that maintaining reindeer corridors in harvest scheduling can be done at minimal cost. Also, we conclude that including corridor constraints in the harvest scheduling model is critical to guarantee connectivity of reindeer pastures.

Keywords: mixed-integer programming; forest management; wildlife conservation; reindeer husbandry; spatial optimization

\section{Introduction}


In Northern Sweden, two distinct industries use the same forestland as a common resource: commercial forestry and the reindeer husbandry.

Reindeer husbandry is central to the livelihood and cultural identity of the Sami, the indigenous people of Sweden. The Sami have practiced reindeer husbandry for over 400 years (Lundmark 2007). As stated by Sandström et al. (2003), "The importance of reindeer husbandry for the Sami cannot be overemphasized". Today $20 \%$ of Sami in Sweden are actively involved in reindeer husbandry through 51 herding communities (Rural Development Programme for Sweden 2008). Approximately half of the productive forest lands in Sweden lay within the reindeer husbandry area (Sandström et al. 2016). Although it is the landowner that has the rights to harvest trees commercially the Sami have legal permission to graze their herds on any land regardless of ownership (SFS1993:36) Reindeer (Rangifer tarandus) are highly migratory, and require vast tracts of land for habitat. During the summer months, reindeer use the mountainous western region of Sweden. However, as temperatures decrease and snow falls, lichen and other vegetation tied to reindeer habitat become inaccessible. As a result, the Sami herders move their herds east towards the coast, where the climate is milder and snow conditions are usually more suitable. After winter, the herders move the reindeer back towards the mountains to their calving grounds in the foothills. This annual migration can cross up to $500 \mathrm{~km}$ each way mostly through forestland (Sandström et al. 2003). During winter and early spring, almost $80 \%$ of the reindeer's diet consists of lichen (Heggeberget et al. 2002). Ground lichen (mostly Cladina spp.) is the primary food source for reindeer in winter, but if snow layers on the ground harden, they rely heavily on arboreal lichen (mainly Bryoria spp.) for food (Bostedt et al. 2003, Kumpula 2001). 
Forestry practices have drastically reduced the amount and accessibility of lichen in northern forests, and many studies have concluded that current forest practices have been detrimental to reindeer husbandry (Berg et al. 2008, Kivinen et al. 2010, Roturier \& Bergsten 2006, Roturier \& Roué 2009, Sandström et al. 2016, Widmark 2006). Forests abundant in ground lichen have decreased with $71 \%$ since the introduction of modern forest practices around 1955 (Sandström et al. 2016). Specifically, short rotations, soil scarification and dense stands negatively impact lichen presence and accessibility in the forest. Additionally, shortened rotations have reduced the amount of old growth forest in which arboreal lichen grows (Esseen et al. 1996). Soil scarification destroys the ground lichen layer, which can take up to 50 years to grow back (Sundén 2003). Dense forests are less suitable to ground lichen growth and difficult for reindeer to move through (Jonsson Cabrajic et al. 2010, Kivinen et al. 2012). The North American species Contorta (Pinus contorta), also known as lodgepole pine, has also become commercially popular due to its short rotations, but usually grows too dense for reindeer to travel through (SSR 2008). In some areas, this has forced herders to move reindeer via trucks, which is expensive and stressful for the animals. In order for reindeer husbandry to continue, more active forest management for accessible winter habitat is required.

The 1979 Swedish Forestry Act and the Swedish FSC standards dictate that forest owners must account for reindeer husbandry in their timber harvesting proposals. However, it is often unclear as to how much effort foresters are supposed to make. This has been subject to debates for many years. Forest management plans that can satisfy the needs of the herders and prove profitable for forest companies are critical for the welfare of both industries, but are difficult to create. Unfortunately there has been little research into multi-use planning despite this need. Bostedt et al. (2003) considered a 
scenario in which a herder and forester co-manage a forest in order to maximize revenue for both parties. The authors find that joint management greatly improves the revenue for the herders with minimal negative effects on the forester. Korosuo et al. (2014) use long term forest simulations to explore the trade-offs and possible synergies in harvest revenue versus lichen habitat for two forests. They find that current practices are not only suboptimal for harvest revenue, but that they are also devastating for lichen availability. One forestry scenario yielded $2 \%$ higher net present value (NPV) than the "business-as-usual" scenario and more than doubled the amount of lichen for reindeer. The scenario that explicitly considered reindeer husbandry needs decreased NPV by only $5 \%$. The results from both of these studies suggest that collaborative management can result in high gains for reindeer herders at a low cost to foresters.

However, to date, no approach for collaborative planning has incorporated accessibility of lichen resources. Not only does lichen need to be present on the landscape, but it must have easy access for reindeer. In landscape management, wildlife corridors are used to connect habitat for wildlife populations. Wildlife corridors are spatially contiguous linkages across the landscape that facilitate movement and provide access to resources for species in need of protection. Reindeer corridors through managed forests should provide sufficient resources, lichen in particular, for reindeer migrating between summer and winter pastures as well as between grazing areas within a seasonal range. Thus, the challenge is to create a harvest schedule that is economically attractive for foresters while providing high quality corridors for reindeer. One powerful tool for creating harvest schedules as well as selecting wildlife corridors is mathematical programming. Spatially explicit harvesting schedules can be created with mixed-integer programs (MIPs). MIPs assign each management unit a set of management actions (a.k.a. treatment schedule) across a temporal horizon. Given a 
forest landbase, MIPs find optimal management plans with regards to management goals and restrictions. In a typical harvest scheduling MIP, the model finds the plan that maximizes NPV subject to various restrictions, such as maximum clear-cut size (Murray 1999, McDill et al. 2002, Goycoolea et al. 2005, Constantino et al. 2008) and road construction costs (Richards \& Gunn 2000).

Mixed-integer programming could also be useful for creating spatially contiguous areas for wildlife. Given a landscape divided into management units, reserve selection MIPs determine the set of contiguous land parcels that maximize utility for wildlife populations without exceeding a given budget. Graph theory and network optimization techniques are commonly used in contiguous reserve selection models (Williams 1998, Önal \& Briers 2006, Conrad et al. 2012, Jafari \& Hearne 2013). Carvajal et al. (2013) propose a model that incorporates wildlife corridors with harvest scheduling. Their cutting plane approach to connectivity proves computationally tractable for forests up to 1,363 units when planning for 3 time periods.

However, none of these models account for or control corridor geometry. When selecting a corridor from a landscape that is partitioned into irregularly shaped polygons, these models may select a corridor that is too narrow or too long for it to be useful. St John et al. (in review) embeds techniques from artificial intelligence into an MIP approach to control geometric characteristics such as corridor width and length on real landscapes.

In this paper, we demonstrate how St John et al.’s (in review) hybrid artificial intelligence/MIP model can be used to create a harvest schedule for commercial forests that maintains high quality corridors for reindeer herds. For demonstration purposes, we use a commercial forest (Figure 1) [Figure 1 near here] covering approximately 14,000 hectares located in the county of Västernorrland, Sweden, an area actively used 
by reindeer. We investigate whether it is possible to maintain reindeer corridors, and if it is, at what cost to the forest owner in terms of forgone timber revenues.

\section{Material and methods}

\subsection{Corridor Habitat}

In Kivinen et al. (2010), the authors state "Ideal winter grazing areas have a high abundance of both ground growing and arboreal lichens that are easily accessible to reindeer”. This is also true of the routes the reindeer travel in between their winter and summer grazing areas. To be successful, the reindeer corridors need to contain sufficient amounts of both ground and arboreal lichen habitat, and must allow unobstructed travel. Arboreal lichen is typically present in old growth stands (Esseen et al. 1996), so we consider arboreal lichen present in all stands that are older than 120 years old.

Ground lichen grows in managed pine stands with basal area less than $20 \mathrm{~m}^{2} / \mathrm{ha}$ (Dettki and Esseen 1998, Sandström et al 2016). Reindeer avoid grazing in early successional stage forests (Horskotte et al. 2014), thus we assume ground lichen isn’t available for 10 years after a clearcut. If soil scarification occurred, ground lichen has been destroyed and re-establishment can take up to 50 years (Sundén 2003).

Reindeer corridors must have a certain width to facilitate the travel of animals. If the corridor becomes too narrow, it can be difficult to move reindeer herds along. There is no information available on reindeer corridor width requirements, so based off of expert opinion, we set the minimum corridor width at 50 meters in our model.

Corridors also must allow for movement. Some units such as Contorta stands (SSR 2008) are impenetrable for reindeer, thus are excluded from the corridor. Similarly, stands with trees taller than 3 meters with more than 1500 stems/ha and any stand with 
more than 2000 stems/ha are also considered too dense for reindeer movement (SSR 2008)

\subsection{Model}

We use the approach described in St John et al. (in review) to create harvest schedules with reindeer corridors. The approach, called the Optimal Corridor Construction Approach (OCCA), allows us to account for and control the width and length of the corridors in a MIP.

Following the OCCA, we define the polygons of our landscape as 1) individual units (forested and otherwise), and 2) contiguous sets of units whose combined area does not exceed 2 ha. This allows for corridors that can be multiple units wide. For example, in Figure 2, [Figure 2 near here] if the combined area of $p_{j}$ and $p_{k}$ is less than 2 ha, then a corridor may begin at $p_{h}$, go through $p_{j} \cup p_{k}$ and end at $p_{\ell}$.

To calculate the width and length contribution of a polygon to a corridor, we must determine 1) where the corridor's route is entering/exiting the polygon and 2) how the route moves through the polygon. For example, in Figure 2, the route from $p_{i}$ through $p_{k}$ to $p_{j}$ will have a different width than the route from $p_{h}$ through $p_{k}$ to $p_{\ell}$. We represented the pairs of entrance/exit points with gate pairs, denoted by $(m, n)$ where $m$ and $n$ are gates that serve as transition points between the current polygon and adjacent polygons. For each gate pair, there may be many possible routes through its associate polygon. In Figure 2, there are two routes associated with the route from gate $\boldsymbol{m}_{\mathbf{0}}$ through $p_{h}$ to gate $\boldsymbol{n}_{\mathbf{0}}$. To determine the width and length of the route from gate $m$ through $p$ to gate $n$ of maximal width, we solve a preprocessing MIP, identical to that in St John et al. (in review). The preprocessing MIP is network flow problem, in which we find the route from gate $m$ to gate $n$ of maximal width. If the width of 


\section{the optimal route meets the corridor width requirement, then the gate pair is}

\section{eligible to be in the corridor. Let $G$ be the set of all such gate pairs.}

Finally, we formulate and solve the harvest scheduling MIP. Let $x_{i j}$ be the binary decision variable that takes the value 1 when unit $i$ follows treatment schedule $j$. Let $\beta_{i j}$ be the NPV associated with unit $i$ following treatment schedule $j$. Then, the objective is as follows:

$$
\max \sum_{i, j} \beta_{i j} x_{i j}
$$

The first set of constraints address harvest scheduling logistics. Let $U$ be the set of all units and $J_{i}$ be the set of all possible treatment schedules for unit $i$. Let $h_{i j}^{t}$ be the volume harvested from unit $i$ at time $t$ when following treatment schedule $j$, and let $f$ be the allowable fluctuation in harvest volume from one time period to the next. Then,

$$
\begin{array}{ll}
\sum_{j} x_{i j}=1 & \forall i \in U \\
\sum_{i, j} h_{i j}^{t} x_{i j} \leq(1+f) \sum_{i, j} h_{i j}^{t-1} x_{i j} & \forall t \in\{1,2, \ldots, T\} \\
\sum_{i, j} h_{i j}^{t} x_{i j} \geq(1-f) \sum_{i, j} h_{i j}^{t-1} x_{i j} & \forall t \in\{1,2, \ldots, T\} \\
x_{i j} \in\{0,1\} & \forall i \in U, j \in J_{i}
\end{array}
$$

Constraint (2) requires that only one prescription can be applied to each unit.

Constraints (3) and (4) ensure that the fluctuation of volume harvested in adjacent time periods is less than a predefined bound, $f$. Constraint (5) defines $x_{i j}$ as binary. 
The next set of constraints enforces corridor connectivity. To select our corridor, we modify the transshipment-based model from Jafari and Hearne (2013). This model is introduced as an unrooted fully connected network selection model. Given a landscape of units, Jafari and Hearne's model selects a set of management units for a connected reserve that maximizes the utility of the reserve, subject to budget constraints. Their model uses flow to ensure connectivity. Each unit selected in the reserve has flow entering it, either from another unit in the reserve or by being the initial unit, and "consumes" an amount of flow equal to the cost of including it in the reserve. The total amount of flow allowed is less than or equal to the budget for reserve selection.

We use this model with the St John et al. (in review) graph-theoretical landscape representation. We modify it by creating pseudo-polygons that represent the areas adjacent to the landscape that we wish to connect. In Figure 1, the pseudo-polygons are the shaded polygons. We force these pseudo-polygons to be the first and last units selected for the reserve. Also, we use utility aspects (such as lichen availability) as constraints rather than the objective, and do not restrict the amount of flow.

Let $z_{m n}^{t}$ denote the binary variable that takes the value 1 if gate pair $(m, n)$ is in the corridor at time $t$ and 0 otherwise.

$$
z_{m n}^{t} \in\{0,1\} \quad \forall(m, n) \in G, t \in\{0,1,2, \ldots, T\}
$$

Let $S(G)$ and $E(G)$ be the sets of gate pairs adjacent to the starting and ending pseudo-polygons. We ensure that the corridors begin and end at the pseudo-polygons by requiring one gate pair from $S(G)$ and one gate pair from $E(G)$ to be in the corridor. 


$$
\begin{array}{ll}
\sum_{(m, n) \in S(G)} z_{m n}^{t}=1 & \forall t \in\{0,1,2, \ldots, T\} \\
\sum_{(m, n) \in E(G)} z_{m n}^{t}=1 & \forall t \in\{0,1,2, \ldots, T\}
\end{array}
$$

Let $y_{m n}^{t}$ be the variable that represents the amount of flow crossing gate pair $(m, n)$ at time $t$. We inject flow into the network in Constraint (9). Constraint (10) requires that if a gate pair is selected to be in a corridor, there must be flow entering the gate pair, and it consumes 1 unit of flow.

$$
\begin{aligned}
& \sum_{(m, n) \in S(G)} y_{m n}^{t} \leq|G| \quad \forall t \in\{0,1,2, \ldots, T\} \\
& \sum_{\substack{n: \\
(m, n) \in G}} y_{m n}^{t}-\sum_{\substack{\ell: \\
(\ell, m) \in G}} y_{\ell m}^{t}=\sum_{\substack{n: \\
(m, n) \in G}} z_{m n}^{t} \forall m, t \in\{1,2, \ldots, T\}
\end{aligned}
$$

Next, for every gate $n$, we require that it is selected to be an exit gate of a gate pair $(m, n)$ at most once.

$$
\sum_{m:(m, n) \in G} z_{m n}^{t} \leq 1 \quad \forall n, t \in\{0,1,2, \ldots, T\}
$$

To ensure that $y_{m n}^{t}=0$ if $z_{m n}^{t}=0$, and $0 \leq y_{m n}^{t} \leq|G|$ for all $y_{m n}^{t}$, we include Constraints (12) and (13).

$$
\begin{array}{lr}
y_{m n}^{t} \geq z_{m n}^{t} & \forall(m, n) \in G, t \in\{0,1,2, \ldots, T\} \\
y_{m n}^{t} \leq|G| z_{m n}^{t} & \forall(m, n) \in G, t \in\{0,1,2, \ldots, T\}
\end{array}
$$


Since we are using multiunit polygons, we must include a constraint that ensures the gate pair polygons do not overlap. In Figure 2, a corridor cannot include $p_{j}$ and the polygon $p_{j} \cup p_{k}$. We can combine this with the constraint that does not allow impenetrable units in the corridor. Let $\operatorname{mid}(m, n)$ be the polygon associated with gate pair $(m, n)$ and let $\mathrm{Q}$ be the set of all polygons on the landscape. Also, let $\gamma_{i j}^{t}$ be $\mathrm{a}$ binary coefficient that equals one if unit $i$ is impenetrable at time $t$ when following treatment schedule $j$. Then, Constraint (14) ensures that at most one gate pair associated with polygon $p$ is selected at time $t$, or part of $p$ is impenetrable.

$$
\sum_{\operatorname{mid}(m, n) \cup p \neq \varnothing} z_{m n}^{t}+\sum_{i \in p, j} \gamma_{i j}^{t} x_{i j} \leq 1 \quad \forall p \in P, t \in\{0,1,2, \ldots, T\}
$$

Next in the model, tree and arboreal lichen within the corridor is accounted for and controlled. We define variables $g_{p}^{t}$ and $a_{p}^{t}$ that are equal to the amount of ground and arboreal lichen present in polygon $p$ in hectares if $p$ is in the corridor at time $t$. Otherwise, they are equal to zero. Let $\theta_{i j}^{t}$ and $\phi_{i j}^{t}$ be binary coefficients indicating if ground lichen and arboreal lichen are present in unit $i$ in time $t$ if treatment schedule $j$ is followed and $\lambda_{i}$ be the area of unit $i$.

$$
\begin{array}{cc}
g_{p}^{t} \leq \sum_{i \in p, j \in J_{i}} \lambda_{i} \theta_{i j}^{t} x_{i j} & \forall p \in P, t \in\{0,1,2, \ldots, T\} \\
a_{p}^{t} \leq \sum_{i \in p, j \in J_{i}} \lambda_{i} \phi_{i j}^{t} x_{i j} & \forall p \in P, t \\
\in\{0,1,2, \ldots, T\} &
\end{array}
$$


Constraints (15) and (16) define the upper bound on the amount of ground and arboreal lichen available in a polygon in a time period. The RHS calculates the amount of lichen available in $p$ based on the treatment schedules selected for each unit in the polygon. In Constraints (17), (18) and (19), we ensure that $g_{p}^{t}$ and $a_{p}^{t}$ are nonzero only if $p$ is selected to be in the corridor in time $t$ :

$$
\begin{aligned}
& g_{p}^{t} \leq\left(\sum_{i \in p} \lambda_{i}\right) \sum_{m i d(m, n)=p} z_{m n}^{t} \\
& a_{p}^{t} \leq\left(\sum_{i \in p} \lambda_{i}\right) \sum_{m i d(m, n)=p} z_{m n}^{t} \\
& g_{p}^{t}, a_{p}^{t} \geq 0
\end{aligned}
$$$$
\forall p \in P, t \in\{0,1,2, \ldots, T\}
$$$$
\forall p \in P, t \in\{0,1,2, \ldots, T\}
$$$$
\forall p \in P, t \in\{0,1,2, \ldots, T\}
$$

Lastly, we require that for each time period, the corridor must contain at least $G_{\text {min }}$ hectares of ground lichen and $A_{\text {min }}$ hectares of arboreal lichen.

$$
\begin{array}{ll}
\sum_{p \in P} g_{p}^{t} \geq G_{\min } & \forall t \in\{0,1,2, \ldots, T\} \\
\sum_{p \in P} a_{p}^{t} \geq A_{\min } & \forall t \in\{0,1,2, \ldots, T\}
\end{array}
$$

\subsection{Study area}

Our case study is a 1,996 unit forest owned and managed by Holmen, a Swedish forestry company and used by Vilhelmina Norra reindeer herding community during the prewinter and winter grazing season. The forest is located in an area that is used by 
reindeer herders every winter as they move their herds between their summer and winter pastures as well as for winter grazing. We consider the northern and southern boundaries of the forest (shaded regions in Figure 1) as the regions that we wish to connect with corridors. Along with timberland, areas without productive forest are contained within the forest boundary. We partition these non-forested areas (mostly comprised of lakes, mires, streams, etc.) using ESRI ArcGIS's fishnet tool with a $500 \mathrm{x}$ $500 \mathrm{~m}$ grid. The resulting non-forested units are considered as possible parts of the corridor system (light grey units in Figure 1). The overall planning horizon was set to 50 years and divided into 10 periods of 5 years (i.e. $T$ was set to 10 ). Based on Holmen's 2007 forest inventory data, we generated a set of treatment schedules with future forest conditions (e.g. basal area, number of stems and harvest volumes) for each stand using the Heureka system.

The Heureka system is a comprehensive planning system for multiple-use forestry (Wikström et al 2011). It is based on projections of the tree cover development. Heureka projects future forest states by using data on current conditions, applied management actions, and different functions for e.g. growth and mortality. Forecasts can be made for a large number of variables, including common forest variables such as timber volume, age, species distribution and the output of various products e.g. sawlogs, pulpwood, and biomass.

We generated treatment schedules in the Heureka system in two phases. First, based on its initial conditions, we allocated each stand to one or more management categories (see Table 1). [Table 1 near here] Each management category is associated with different forest activities, such as stand establishment activities, precommercial thinning, thinning, and final felling. Then, for each stand, we generated a set of possible treatment schedules for the 50 year planning horizon based on its associated 
management category. This resulted in an average of 28 treatment schedule alternatives per stand.

Economic data used (timber, regeneration, and harvesting costs) for calculating the NPV for each schedule is based on a timber price list from the current pricelist for the forest owner's organization in northern Sweden. NPVs used a 2.5\% discount rate. In the model, we require that harvested volume in each time period is within $15 \%$ of the volume of the previous time period to ensure that harvesting is relatively consistent across the planning horizon.

\subsection{Computational Experiment}

We used IBM ILOG's CPLEX 12.6 to solve all MIPs, i.e. to select a treatment schedule for each stand. We first solved the model without reindeer corridors for a benchmark to compare the solution to. Next we included the corridors in the model. The 3,823-unit landscape (1,996 forested stands and 1,827 non-forested units) resulted in 4,461 polygons and 120,572 gate pairs. All gate pair MIPs solved to optimality (0\% gap) within 124 minutes total.

To solve the full corridor model, we used a Windows Server 2012 R2 Standard with Intel ${ }^{\circledR}$ Xeon ${ }^{\circledR}$ CPU E5-2680 v2 @ 2.80 GHz (2 processors) with 128 GB RAM and a 64 bit OS. The full model for harvest scheduling with reindeer corridors proved too large to solve in a reasonable amount of time, so we created an initial feasible (but suboptimal) solution to use as a warm start for a reasonable solve time. Our initial solution was found by first finding a harvest schedule that had minimal impenetrable units. We then fixed the schedule, and found a single corridor for all time periods that was not required to meet the ground lichen requirements. Then, we created and solved a MIP for each corridor that maximized ground lichen subject to the fixed harvest schedule, and ran until a corridor that met the ground lichen requirements was found. 
Next, we fixed all of the corridors and found a harvest schedule that maximized NPV while maintaining the feasible corridors found. This resulted in the final feasible solution that was used as a warm start for the full problem, which we ran for 30 hours.

\section{Results}

When no corridors were included in harvest scheduling, the optimal schedule had a NPV of approximately 7,839,000.00 SEK ( $<0.01 \%$ gap). Figures 3 and 4 illustrate the amount of arboreal lichen, ground lichen and impenetrable stands at the/initial forest state and for time periods 3, 7 and 10 if the optimal harvest schedule was followed. [Figures 3 and 4 near here] Over time, the amount of impenetrable forest increases, until it would be impossible for reindeer to move from the northern border to the southern, regardless of corridor width or lichen requirements.

When solving the harvest schedule with corridors problem, the initial feasible solution we set had a starting optimality gap of $3.82 \%$. After running CPLEX for 30 hours, the solution found yielded a harvesting schedule with NPV of approximately $7,579,000.00$ SEK, with an optimality gap of $3.42 \%$. Thus, to include corridors, the amount of NPV forgone is approximately 260,000.00 SEK, or 3.32\%. Table 2 shows the volumes harvested in each time period for the harvest schedules with and without corridors. [Table 2 near here] Although harvest volumes at each time period differ between the two harvest schedules, there is little difference in the overall harvested volumes.

The corridor conditions for each time period are described in Table 3. [Table 3 near here] Maps showing the corridors, lichen and penetrability of the forest for the initial landscape and in time periods 3, 7 and 10 are shown in Figures 5 and 6. [Figures 5 and 6 near here] The solution found different corridors for each time period, but all corridors satisfy the requirements related to corridor quality. With the exception of time 
period 3, the amount of ground lichen within the corridor decreased over time. Arboreal lichen increased over time, starting at 53 ha and ending with over 320 ha within the corridor. Corridor length was not restricted, so in some cases corridors were long and winding in order to meet lichen requirements (see Figure 5 Time Period 3 for example). For example, the corridor for time period 9 is nearly double the length of the corridor in time period 1. Also, the corridor for time period 10 touches the northern forest boundary at the beginning of the corridor and in the middle (see Figure 6). The amount of impenetrable forest across the landscape increases over time (see Figures 5 and 6), but the corridors guarantee reindeer can cross the forest from the northern boundary to the southern throughout the 50 year planning horizon.

\section{Discussion and Conclusions}

In this paper, we have shown that it is possible to incorporate high quality reindeer corridors into a harvest schedule with a small effect on the commercial production of the forest. Since the corridors only directly affect a small portion of the forest, the cost of their inclusion is also relatively low. For instance, in Korosuo et al. (2014), the NPV forgone in order to maximize lichen retention on the landscape was 5\%, compared to the 3.42\% foregone to include corridors. Meanwhile, we ensure connectivity of the landscape for the reindeer over the planning horizon. These findings offer a possibility for a more comprehensive way to ensure viable conditions for reindeer husbandry when planning forest management.

In both harvest schedule scenarios, there is a dramatic increase in the amount of impenetrable forest by the end of the planning horizon (see Figures 3 through 6). By time period 10, both forests are almost completely impenetrable. However, in the harvest schedule with corridors, there exists at least one path through the forest that meets the needs of the reindeer. In the harvest schedule that did not consider corridors, 
it is impossible to traverse the forest from the northern boundary to the southern. Thus, we conclude that including corridor constraints in the harvest scheduling model is critical to guarantee reindeer passage.

When finding a harvest schedule with corridors, computational tractability proved to be difficult to achieve. Only by incrementally building up a feasible solution, and using a powerful server, we were able to solve the full problem to within a reasonable optimality gap. Even so, after running the full problem for 30 hours, the gap only improved by $0.40 \%$. Running CPLEX with a "good” initial feasible solution seems critical to finding a near optimal solution. Further research into the underlying causes and ways to improve computational performance is necessary in understanding why incorporating corridors makes the problem nearly intractable.

In this paper, we demonstrated our approach to incorporate reindeer corridors in a harvest scheduling model using a specific case study. This method also can be applied to any scenario involving maintenance of high quality corridors across time. With this approach we can control geometric characteristics (e.g. length, width, orientation, turn angle) as well as ecological characteristics (e.g. food, shelter, accessibility) of corridors. For our case study we found corridors that satisfy a list of requirements, but it would be straightforward to find corridors that maximize or minimize some characteristic, and also to find corridors that improve over time. For example, we can maximize the amount of ground lichen in the corridor, or require that the width of the corridor increases over time. This approach can also be extended to other scenarios in which "paths" are selected on a landscape, such as including fuel breaks in harvest scheduling, or selecting units to create hiking trails.

We have shown with a real case study that it is possible to create spatially explicit forest management plans in which the needs of the reindeer are satisfied, but 
commercial operations are still maximized. Historically, finding solutions that both herders and foresters are satisfied with has been extremely challenging. However, with state-of-the-art mathematical programming tools, solutions can be found that satisfy both parties with minimal compromise. In situations such as that of the conflict between the Sami reindeer herders and the commercial forest owners, it is possible to find real and practical solutions in which both parties are satisfied.

\section{Acknowledgements}

This project was funded jointly by the Swedish University of Agricultural Sciences and the Precision Forestry Cooperative at the University of Washington. The work was also partially financed by the EU FP7 project Advanced multifunctional management of European mountain forests (ARANGE). We would also like to thank Holmen for the forest dataset.

\section{References}

Berg A, Östlund L, Moen J, Olofsson J. 2008. A century of logging and forestry in a reindeer herding area in northern Sweden. For Ecol Manag. 256:1009-1020.

Bostedt G, Parks P, Boman M. 2003. Integrated natural resource management in northern Sweden: an application to forestry and reindeer husbandry. Land Econ. 79:149-159.

Carvajal R, Constantino M, Goycoolea M, Vielma JP, Weintraub A. 2013. Imposing connectivity constraints in forest planning models. Oper Res. 61:824-836.

Conrad J, Gomes C, van Hoeve WJ, Sabharwal A, Suter J. 2012. Wildlife corridors as a connected subgraph problem. J of Environ Econ Manag. 63:1-18. 
Constantino M, Martins I, Borges J. 2008. A new mixed-integer programming model for harvest scheduling subject to maximum area restrictions. Oper Res. 56:542-551. CPLEX, IBM ILOG. 2011. http://www.ibm.com/software/integration/optimization/ cplex/.

Dettki H, Esseen PA. 1998. Epiphytic macrolichens in managed and natural forest landscapes: a comparison at two spatial scales. Ecography. 21:613-624.

Esseen PA, Renhorn KE, Petterson R. 1996. Epiphytic lichen biomass in managed and old-growth boreal forests: effect of branch quality. Ecol Appl. 6:228-238.

Goycoolea M, Murray A, Barahona F, Epstein R, Weintraub A. 2005. Harvest scheduling subject to maximum area restrictions: exploring exact approaches. Oper Res. 53:490-500.

Heggeberget T, Gaare E, Ball J. 2002. Reindeer (Rangifer tarandus) and climate change: importance of winter forage. Rangifer. 22:13-22.

Horstkotte, T., Sandström, C., \& Moen, J. (2014). Exploring the multiple use of boreal landscapes in northern Sweden. the importance of social-ecological diversity for mobility and flexibility. Hum Ecol, 42(5), 671-682.

Jafari N, Hearne J. 2013. A new method to solve the fully connected Reserve Network Design Problem. Eur J Oper Res 231:202-209.

Jonsson Cabrajic, A.V., J. Moen, and K. Palmqvist. 2010. Predicting growth of mat-forming lichens on a landscape scale-Comparing models with different complexities. Ecography 33: 949-960.

Kivinen S, Berg A, Moen J, Östlund L, Olofsson J. 2012. Forest fragmentation and landscape transformation in a reindeer husbandry area in Sweden. Environ Manag. 49:295-304. 
Kivinen S, Moen J, Berg A, Eriksson Å. 2010. Effects of modern forest management on winter grazing resources for reindeer in Sweden. Ambio 39:269-278.

Korosuo A, Sandström P, Öhman K, Erikkson LO. 2014. Impacts of different forest management scenarios on forestry and reindeer husbandry. Scand J For Res. 29 sup1:234-251.

Kumpula J. 2001. Winter grazing of reindeer in woodland lichen pasture: effect of lichen availability on the condition of reindeer. Small Ruminant Res. 39:121-130. Lundmark L. 2007. Reindeer pastoralism in Sweden 1550-1950. Rangifer. 27:9-16. McDill M, Rebain S, Braze J. 2002. Harvest scheduling with area-based adjacency constraints. Forest Sci. 48:631-642.

Murray A. 1999. Spatial restrictions in harvest scheduling. For Sci. 45:45-52.

Önal H, Briers R. 2006. Optimal selection of a connected reserve network. Oper Res. 54:379-388.

Rural Development Programme for Sweden. 2008. Swedish Ministry of Agriculture. Article no. Jo 08.008.

Richards W, Gunn A. 2000. A model and tabu search method to optimize stand harvest and road construction schedules. For Sci. 46:188-203.

Roturier S, Bergsten U. 2006. Influence of soil scarification on reindeer foraging and damage to planted Pinus sylvestris seedlings. Scand J For Res. 21:209-220. Roturier S, Roué M. 2009. Of forest, snow and lichen: Sami reindeer herders' knowledge of winter pastures in northern Sweden. For Ecol Manag. 258:1960-1967. Sandström C, Mone J, Widmark C, Danell Ö. 2006. Progressing toward comanagement through collaborative learning: forestry and reindeer husbandry in dialogue. Int J Biodiv Sci Manag. 2:326-333. 
Sandström P, Cory N, Svensson J, Hedenås H, Jougda L, Brochert N. (2016). On the decline of ground lichen forests in the Swedish boreal landscape - implications for reindeer husbandry and sustainable forest management. Ambio 1-15. Sandström P, Granqvist Pahlén T, Edenius L, Tømmervik H, Hagner O, Hemberg L, Olsson H, Baer K, Stenlund T, Göran Brandt L. 2003. Conflict resolution by participatory management: remote sensing and GIS as tools for communicating land-use needs for reindeer herding in northern Sweden. AMBIO. 32:557-567. SFS (1993). Rennäringslagen (1993:36). [The law regulating reindeer husbandry]. SFS 1971:437. [In Swedish].

St John R, Tóth S, Zabinsky Z. in review. Combining path planning from artificial intelligence with mixed-integer programming to optimize the geometry of wildlife corridors in reserve design. Oper Res. in review.

Sundén M. 2003. Re-establishment rate of reindeer lichen (Cladina spp.) after soil scarification in Scots pine-lichen forest types in boreal Sweden. Thesis, Forest Sciences, Swedish University of Agricultural Sciences.

[SSR] Svenska Samarnas Riksförbund. 2008. Ett renskötselanpassat skogsbruk [Reindeer husbandry-adapted forestry] [Internet]. Umeå: Svenska Samernas Riksförbund. [cited 2015 Nov 18]. Available from: http://www.sapmi.se/skogspolicy.pdf Widmark C. 2006. Forestry and reindeer husbandry in northern Sweden- the development of a land use conflict. Rangifer. 26:43-54.

Wikström P, Edenius L, Elfving B, Eriksson, LO, Lämås T, Sonesson J, Öhman K, Wallerman J, Waller C, Klintebäck F. 2011. The heureka forestry decision support system: an overview. MCFNS. 3:87-95. 
Williams J. 1998. Delineating protected wildlife corridors with multi-objective programming. Environ Model Assess. 3:77-86.

Table 1. The allocation of possible management category for the stands depending on initial condition

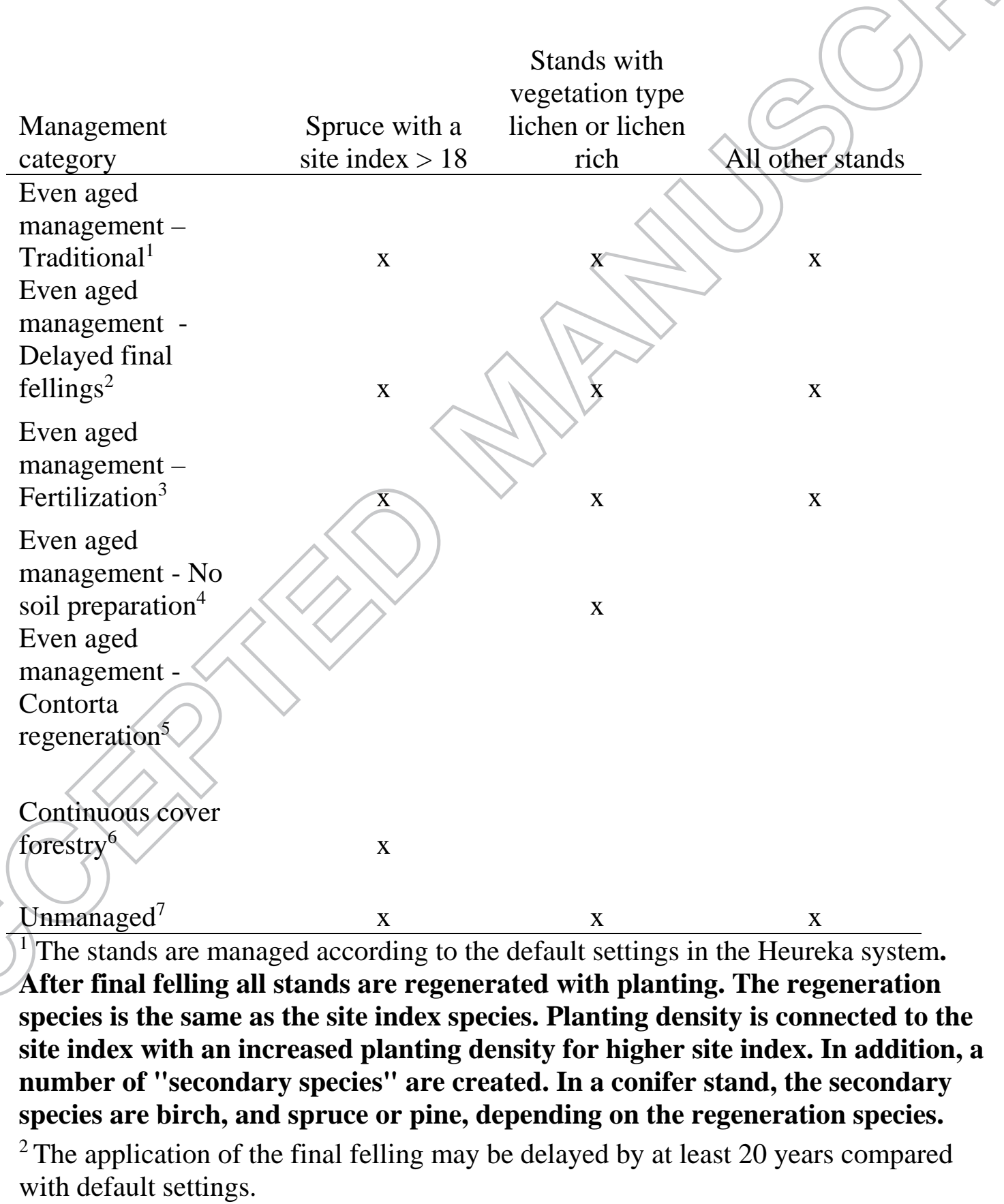


${ }^{3}$ The stands are fertilized 10 years before final felling in addition to the default settings

${ }^{4}$ No soil preparation is allowed after final felling

${ }^{5}$ The stands are generated with contorta after final felling

${ }^{6}$ The stands are subject to selective cuttings with at least 20 years between cuttings

${ }^{7}$ The stands are left unmanaged during the planning horizon

Table 2. Harvest volumes in thousand $\mathrm{m}^{3}$ across planning horizon for each harvesting schedule for 5 year long time periods.

\begin{tabular}{ccc}
\hline Time period & Schedule with no corridors & Schedule with corridors \\
\hline 1 & 66.019 & 58.789 \\
2 & 56.117 & 49.996 \\
3 & 47.700 & 42.504 \\
4 & 40.548 & 36.164 \\
5 & 34.471 & 30.760 \\
6 & 29.305 & 26.150 \\
7 & 24.917 & 22.228 \\
8 & 21.178 & 23.959 \\
9 & 18.005 & 27.076 \\
10 & 15.307 & 31.102 \\
\hline Total & 353.571 & 348.728 \\
\hline
\end{tabular}


Table 3. Information on corridors selected by model.

\begin{tabular}{ccccc} 
Time Period & $\begin{array}{c}\text { Ground } \\
\text { Lichen (ha) }\end{array}$ & $\begin{array}{c}\text { Arboreal } \\
\text { Lichen (ha) }\end{array}$ & $\begin{array}{c}\text { Minimum } \\
\text { Width (m) }\end{array}$ & Length (km) \\
\hline 0 & 248.81 & 53.45 & 51.93 & 30.91 \\
1 & 147.30 & 69.04 & 50.41 & 26.10 \\
2 & 167.53 & 75.00 & 50.92 & 35.04 \\
3 & 251.83 & 50.66 & 50.92 & 43.34 \\
4 & 148.18 & 124.35 & 50.64 & 35.00 \\
5 & 144.52 & 238.99 & 51.42 & 38.49 \\
6 & 111.11 & 289.05 & 51.42 & 41.76 \\
7 & 118.23 & 361.03 & 50.58 & 40.94 \\
8 & 105.53 & 346.55 & 50.00 & 43.38 \\
9 & 103.09 & 415.29 & 50.90 & 51.49 \\
10 & 102.43 & 324.77 & 51.34 & 46.56 \\
\hline
\end{tabular}



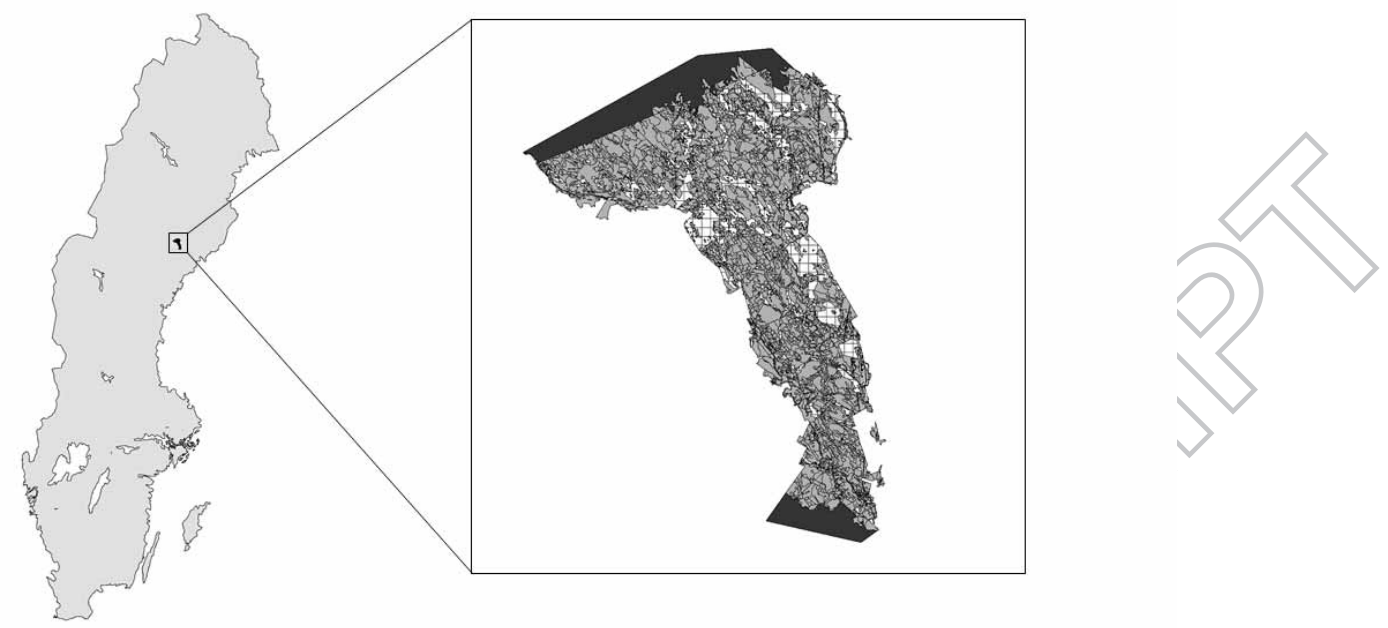

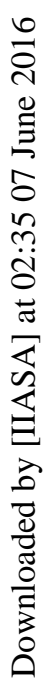



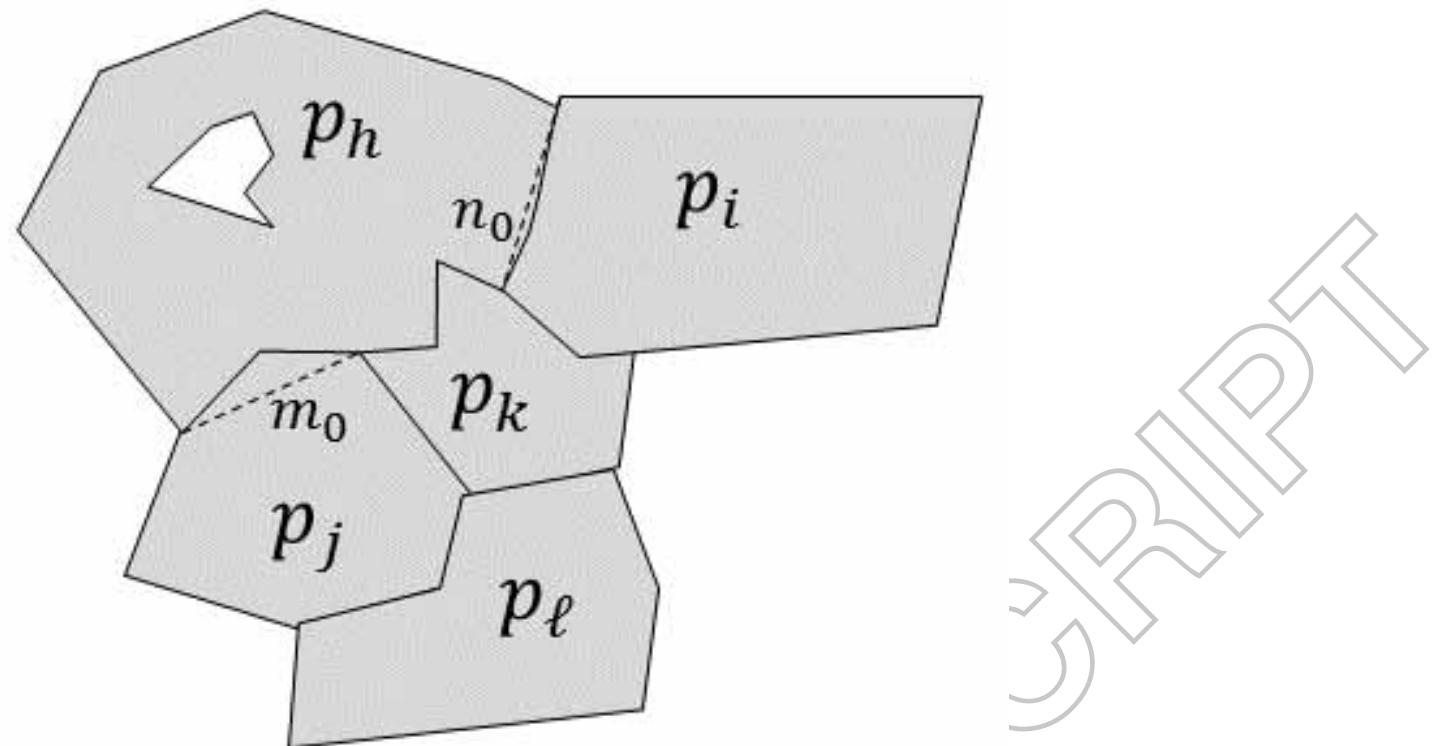

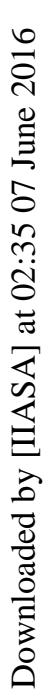



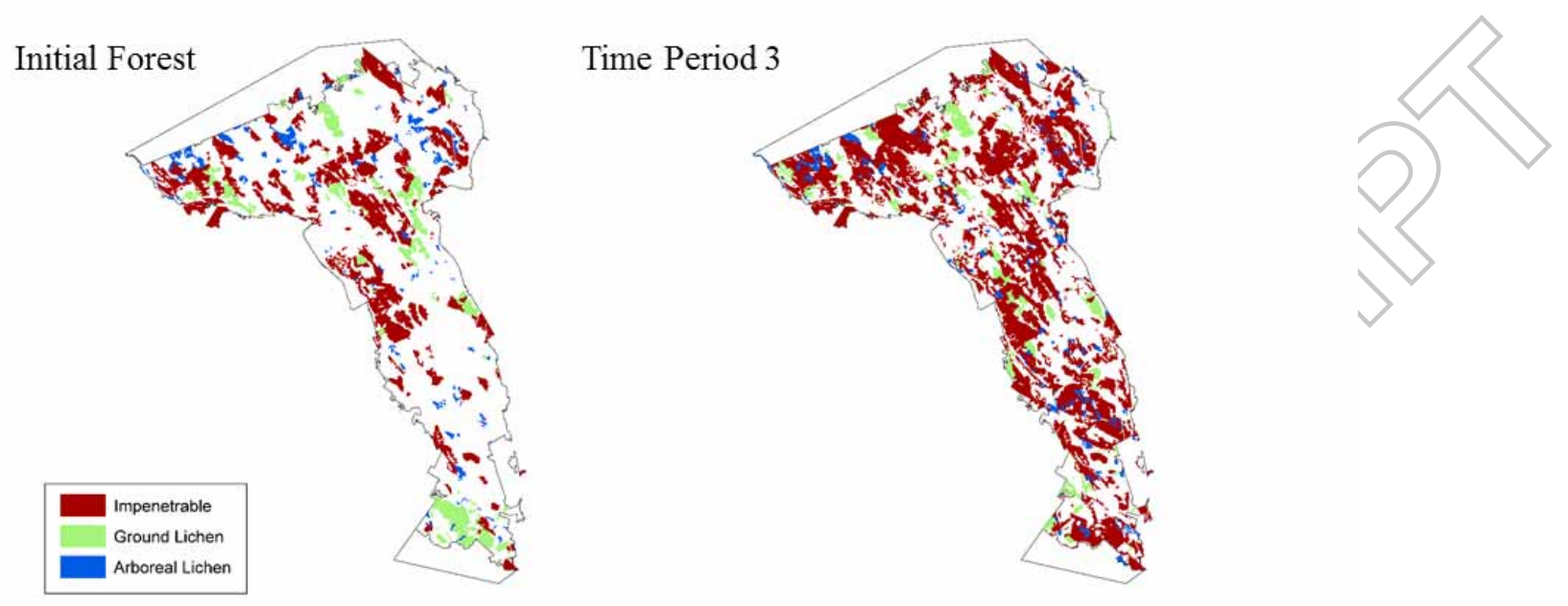

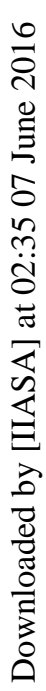



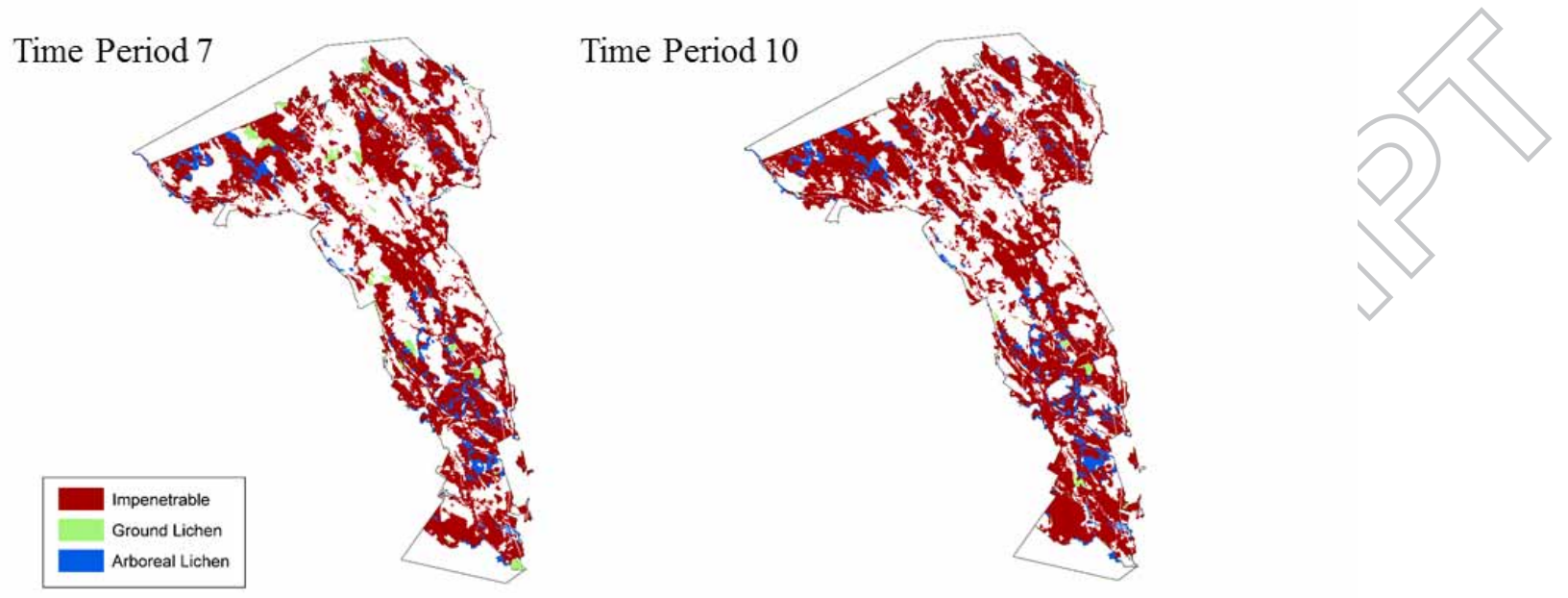


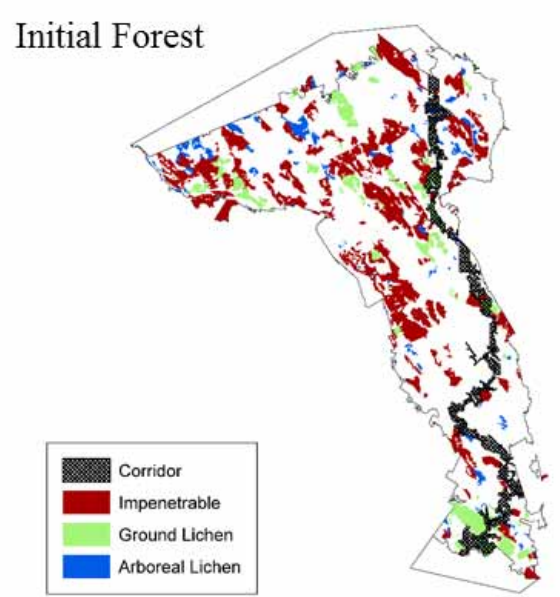

Time Period 3
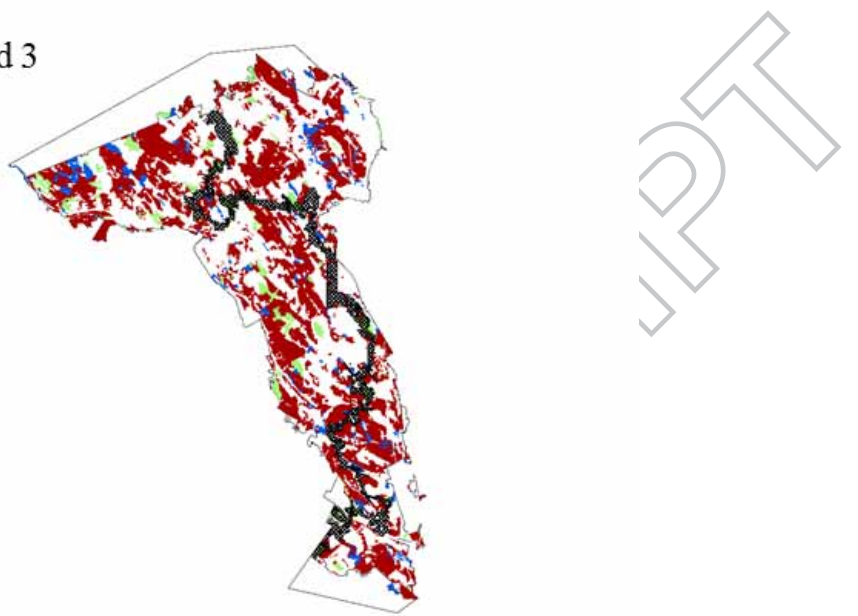

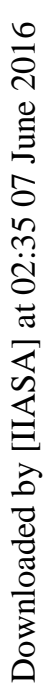



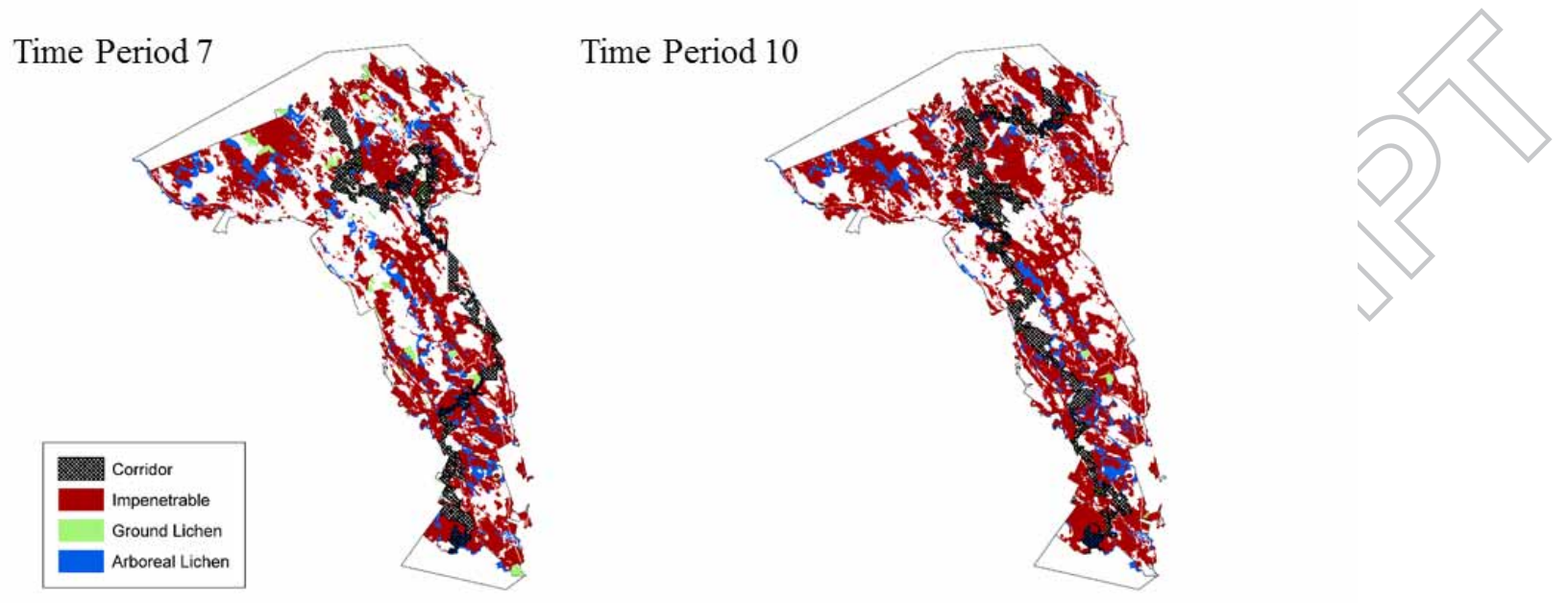